\title{
Avaliação do reparo ósseo por microtomografia por raio-X
}

\author{
Fracture healing valuation using X-ray microtomography
}

\author{
Márcia Regina Milanetti ${ }^{1}$, José Marcos Alves², \\ Alessandro Hakme da Silva', Simone Orlandi Introini'
} Milanetti MR, Alves JM, Silva AH, Introini SO. Avaliação do reparo ósseo por microtomografia por raio-X / Fracutre healing valuation using
X-ray microtomography. Rev Med (São Paulo). 2011 abr.-jun.;90(2):89-93.

RESUMO: A microtomografia 3D por raio-X proporciona medidas quantitativas e tridimensionais da estrutura do calo e essas medidas podem potencialmente estar relacionadas com a resistência do calo. A avaliação quantitativa do reparo ósseo por meio de novas metodologias tem importante aplicação nas pesquisas experimentais relacionadas a tecnologias invasivas e não invasivas para a estimulação do mesmo. O objetivo desse estudo foi avaliar o reparo ósseo por meio da $\mu \mathrm{CT}$ em defeito ósseo em fêmur de rato. Trinta ratos machos da classe Wistar com peso médio de $300 \mathrm{~g}$ foram divididos em grupos experimentais de 10 animais em cada grupo. Os animais foram anestesiados e um furo com $1,2 \mathrm{~mm}$ de diâmetro foi realizada na porção medial

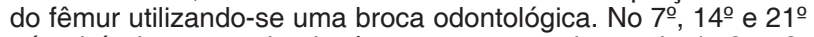
pós-cirúrgico, os animais dos grupos experimentais 1,2 e 3 , respectivamente, foram sacrificados e o fêmur esquerdo excisado. Os fêmures foram envolvidos em gaze e mergulhas em solução PBS e armazenados em um saco plástico em freezer a $-20^{\circ}$ até a análise microtomográfica. Os fêmures foram escaneados pelo microtomógrafo 1172 (SkyScan, Bélgica). Os softwares NRecon, Dataviewer, CT-Analyzer and CT-Vol, fornecidos pelo fabricante do microtomógrafo, foram utilizados para as seguinte análises: a) análise visual das reconstruções microtomográficas dos fêmures através de secções transversais, coronais e sagitais; b) segmentação do calo ósseo nas reconstruções através de algoritmo de processamento de imagem para quantificação dos parâmetros volume total do calo ósseo (TV), volume do calo ósseo mineralizado (BV), relação BV/TV e densidade mineral óssea volumétrica do calo ósseo (BMD); c) visualização 3D do calo ósseo. A análise estatística dos parâmetros medidos utilizou o teste $t$ de Student com um nível de significância $p<0,05$. Houve um aumento estatisticamente significante nos valores dos parâmetros BV/TV e BMD na comparação entre os grupos experimentais 1 e 2 . A comparação entre os grupos 3 e 4 não apresentou significância estatística. Os resultados são coerentes com dados encontrados na literatura sobre a fisiologia óssea, porém o algoritmo de processamento de imagem utilizado necessita aprimoramento em alguns de seus procedimentos para se obter melhor resultado de segmentação do calo ósseo na região de interesse.

DESCRITORES: Calo ósseo/radiografia; Calo ósseo/fisiologia; Microtomografia por raio-X; Osso e ossos/radiografia; Osso e ossos/lesões; Raios-X; Ratos Wistar.
ABSTRACT: X-ray microtomography (UCT) provides quantitative and three dimensional measurements of the callus structure and these measurements could potentially be related to callus strength. The assessment of bone repair through new methodologies has important application in animal investigations regarding invasive or non invasive technologies for the stimulation of bone healing. The aim of this investigation was the use of $\mu \mathrm{CT}$ for the assessment of bone repair in a rat femur bone defect. Thirty male Wistar rats weighting about $300 \mathrm{~g}$ were divided in three experimental groups with 10 animals on each group. The animals were anesthetized and a circular hole with a $1.2 \mathrm{~mm}$ diameter was generated at the medial region of the left femur using a dental drill. At the 7th, 14th and $21^{\text {st }}$ day after surgery the animals of experimental groups 1,2 and 3 , respectively, were sacrificed and the left femur excised. The femurs were wrapped in gauze immersed in phosphate-buffered solution and stored in a plastic bag at $-20^{\circ} \mathrm{C}$ until the analysis by microtomograph. The femurs were scanned by the 1172 microtomograph (SkyScan, Belgium). The softwares NRecon, Dataviewer, CT-Analyzer and CT-Vol, provided by the microtomograph manufacture, were used for the following assessments: a) visual examination of the femurs microtomographic reconstructions using transversal, coronal and sagittal sections; b) segmentation of the bone callus in the reconstructions using an image processing algorithm to quantify the parameters total bone callus volume (TV), volume of the mineralized bone callus (BV), the ratio BV/ TV and the volumetric bone callus mineral density (BMD); c) $3 \mathrm{D}$ rendering of the bone callus. The statistical analysis of the measured parameters was performed by the Student $t$ test with a level of significance $p<0.05$. There was a statistically significant increase in the mean values of the parameters BV/TV and BMD in the comparison of experimental groups 1 and 2 . The comparison between groups 2 and 3 was not statistically significant. Results are according to bone physiology data from literature although the image processing algorithm used needs some adjustments to get better results in bone callus segmentation in ROI.

KEYWORDS: Bony callus/radiography; Bony callus/phisiology; $\mathrm{X}$-ray microtomography; Bone and bones/radiography; Bone and bones/injuries; X-ray; Rats, Wistar.

Trabalho decorrente do mestrado realizado no Departamento de Bioengenharia da Escola de Engenharia de São Carlos da Universidade de São Paulo (EESC-USP), apresentado e publicado nos Anais do XXII Congresso Brasileiro de Engenharia Biomédica, Tiradentes, MG, 2010.

1 Programa de Pós-Graduação Interunidades Bioengenharia - USP. Escola de Engenharia de São Carlos, Faculdade de Medicina de Ribeirão Preto, Instituto de Química de São Carlos, São Carlos, SP, Brasil.

2 Programa de Pós-Graduação Interunidades Bioengenharia - USP, Éscola de Engenharia de São Carlos, Departamento de Engenharia Elétrica, São Carlos, SP, Brasil.

Endereço para correspondência: Márcia Regina Milanetti. e-mail: mrmila@sc.usp.br 


\section{INTRODUÇÃO}

s fraturas ós seas a fe ta m
economicamente a sociedade tanto nos
custosem saúde comona produtividade ${ }^{1}$. A consolidação da fratura é um processo que envolve uma série de eventos celulares e moleculares que obedecem a uma seqüência específica no tempo². Há grande interesse em pesquisas que esclareçam os mecanismos envolvidos no reparo ósseo e que possibilitem o desenvolvimento de técnicas de intervenção que possam acelerar esse processo.

O monitoramento do reparo ósseo em fraturas experimentais, com o objetivo de investigar novas técnicas de tratamento de fraturas, é usualmente realizado por meio de procedimento radiográfico que associado a histomorfometria, imunohistoquímica, ultramicroscopia e ensaios mecânicos possibilitam o entendimento das alterações no tecido ósseo. A análise por microtomografia, que resulta em imagens e quantificações tridimensionais, caracteriza um novo procedimento para o estudo dos eventos que resultam na regeneração óssea ${ }^{3,4}$.

A análise radiográfica é uma avaliação bidimensional da estrutura tridimensional do tecido de reparo ósseo. Morgan e colaboradores ${ }^{5}$ afirmam que a microtomografia por raio-x $(\mu \mathrm{CT})$ proporciona medidas quantitativas e tridimensionais da estrutura e mineralização do calo ósseo e essas medidas podem estar relacionadas a rigidez e resistência mecânica do calo.

Beleville et al. ${ }^{6}$ em um estudo com camundongos monitoraram o reparo do tecido ósseo em fraturas diafisárias de fêmur tratadas com hormônio paratireóide (PTH). Análises tridimensionais do osso fraturado foram realizadas pelo software fornecido pelo fabricante do microtomógrafo e foi possível separar o calo ósseo do tecido adjacente para a medida do volume e superfície óssea do calo formado.

Bakker et al. ${ }^{7}$ monitoraram durante 90 dias a taxa de reparo do osso cortical em um defeito produzido na vértebra da cauda de camundongos utilizando microtomografia in vivo.

A investigação a seguir descrita teve o objetivo de desenvolver uma metodologia de avaliação do reparo ósseo em osteotomia produzida por uma fresa acoplada à uma caneta de alta rotação no terço medial de fêmur de rato utilizando-se $\mu \mathrm{CT}$. A metodologia envolve procedimentos de segmentação, visualização bidimensional (2D) e tridimensional (3D) e análise morfométrica do reparo ósseo formado na osteotomia. Os procedimentos de segmentação e visualização foram implementados e os resultados são apresentados.

\section{MATERIAIS E MÉTODOS}

Os procedimentos experimentais com os animais foram aprovados pelo "Comitê de Ética em Experimentação Animal“ (protocolo 65/2009) da Universidade Federal de São Carlos e executados no Programa de Pós-Graduação Interunidades Bioengenharia - USP (PPGIB-USP).

\section{Animais}

Foram utilizados 30 ratos machos da raça Wistar. Durante o experimento os animais permaneceram em ambiente com ciclo claro-escuro de 12 horas e temperatura controlada. Os animais receberam alimentação composta pela dieta balanceada comercial e água ad libitum.

\section{Cirurgia}

Aos animais, foi administrada uma mistura de $0,1 \mathrm{ml}$ do anestésico cloridrato de cetamina (Ketamina ou Ketamin) e 0,1 $\mathrm{ml}$ do relaxante muscular cloridrato de xilazina (Rompun) para cada $100 \mathrm{~g}$ de massa corporal. A área da cirurgia foi submetida à tricotomia pré-operatória e com o auxilio de um bisturi foi exposto o fêmur direito. Uma osteotomia parcial transversal, seguindo modelo de Lirani [8], foi realizada no terço médio do fêmur direito para se produzir um defeito ósseo na cortical lateral utilizando-se um aparelho da marca Driller K® modelo BLM 600 plus, com caneta de alta rotação (690 rpm) com fresa de $1 \mathrm{~mm}$ de espessura, irrigada com soro fisiológico estéril para controle da temperatura (Figura 1). A sutura da incisão na pele utilizou mono nylon 5.0.

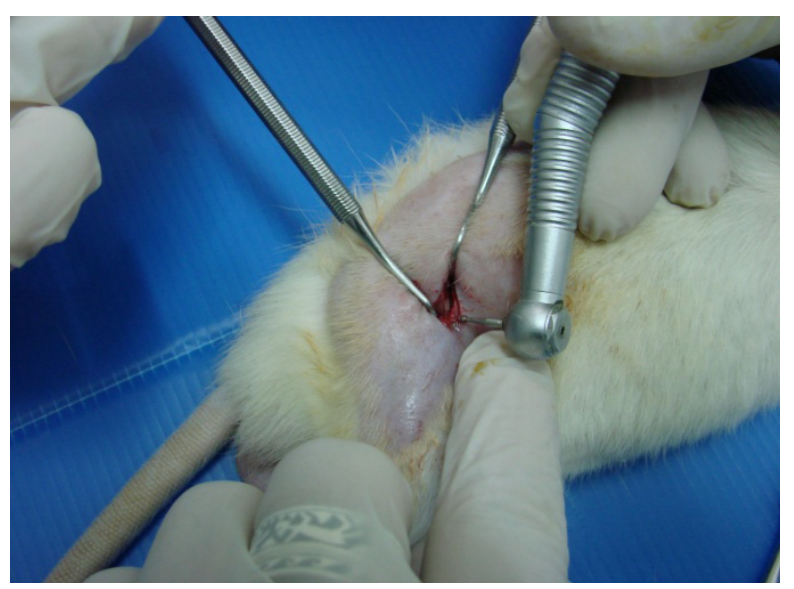

Figura 1. Procedimento cirúrgico para produzir osteotomia na região medial do fêmur com fresa 
Milanetti et al. Avaliação do reparo ósseo por microtomografia por raio-X.

\section{Grupos experimentais}

Os animais foram divididos em três grupos experimentais com 10 ratos em cada grupo. O sacrifício dos animais dos Grupos 1, 2 e 3 ocorreu no $7^{\circ}, 14^{\circ} \mathrm{e}$ $21^{\circ}$ dia após a cirurgia. Os animais foram sacrificados por overdose de Ketamin e Rompun. Os fêmures excisados dos animais foram envolvidos por uma gaze umedecida em uma solução tampão de fosfato (PBS - phosphate buffered solution) e mantidos à $-20^{\circ} \mathrm{C}$ em freezer até a realização dos procedimentos de avaliação do reparo ósseo por UCT.

\section{Avaliação do reparo ósseo por microtomografia}

A avaliação do reparo na osteotomia dos fêmures por UCT foi realizada na Embrapa Instrumentação Agropecuária (São Carlos, SP) utilizando-se o microtomógrafo modelo 1172 (Skyscan, Bélgica) e os softwares NRecon, Dataviewer, CT-Analyzer e CTVol fornecidos pelo fabricante do microtomógrafo. A avaliação compreendeu as seguintes etapas:

1) Escaneamento microtomográfico dos fêmures excisados com os seguintes parâmetros: fonte de raio-X operando com $89 \mathrm{KV}$ e $112 \mathrm{uA}$, filtro de alumínio de $0,5 \mathrm{~mm}$ de espessura, rotação de $180^{\circ}$ com incremento angular de $0,4^{\circ}$, aquisição com câmara CCD de $10 \mathrm{Mp}$ de 8 projeções radiográficas em cada rotação e resolução de 4,7 $\mu \mathrm{m}$;

2) Reconstrução microtomográfica dos fêmures pelo software NRecon utilizando-se o algorítimo de Feldkamp ${ }^{9}$;

3) Visualização coronal, transversal e sagital de reconstruções microtomográficas dos fêmures através do software Dataviewer;

4) Segmentação e visualização $2 D$ do tecido de reparo ósseo nas reconstruções microtomográficas de cada fêmur através do software CT-Analyzer utilizando um algorítimo de processamento de imagem com os seguintes procedimentos: a) thresholding para binarização das reconstruções microtomográficas; b) delimitação da região de interesse contendo o tecido de reparo e o tecido adjacente; c) segmentação do tecido de reparo do tecido adjacente com operações morfológicas de abertura e fechamento;

5) Visualização 3D do tecido de reparo e da região medial dos fêmures contendo o tecido de reparo através do software CT-Vol.

\section{RESULTADOS}

A Figura 2 mostra em diferentes planos de visualização as reconstruções microtomográficas de um fêmur onde se observa o tecido de reparo ósseo na osteotomia produzida no terço medial.
As Figuras 3 à 8 exemplificam em uma reconstrução microtomográfica transversal de um fêmur, após 7 dias de reparo ósseo, o resultado dos procedimentos de thresholding, delimitação da região de interesse, segmentação do tecido de reparo do tecido adjacente e a visualização 3D do tecido de reparo e da região medial do fêmur contendo o tecido de reparo.

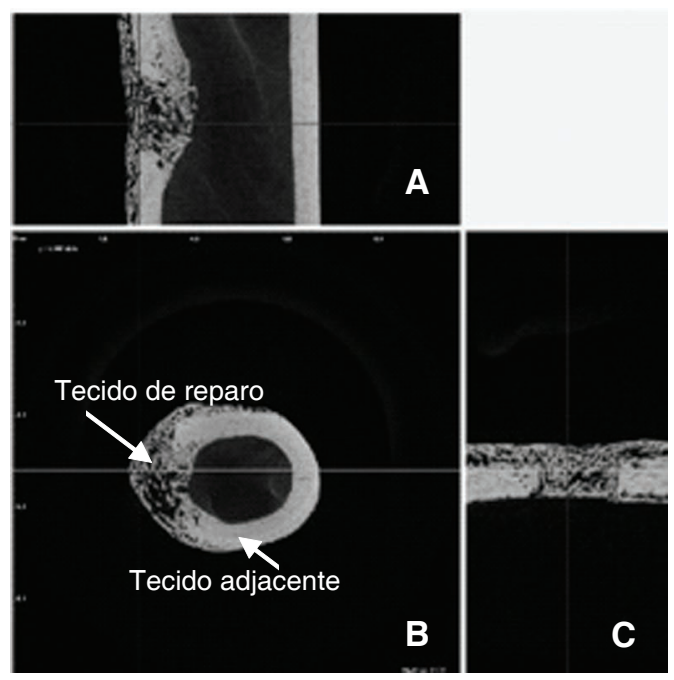

Figura 2. Visualização coronal (A), transversal (B) e sagital (C) das reconstruções microtomográficas de um fêmur

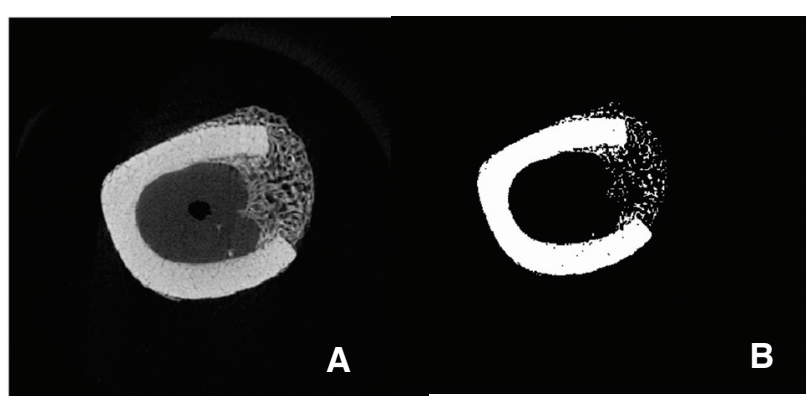

Figura 3. Thresholding (B) de reconstrução microtomográfica transversal em tons de cinza $(A)$

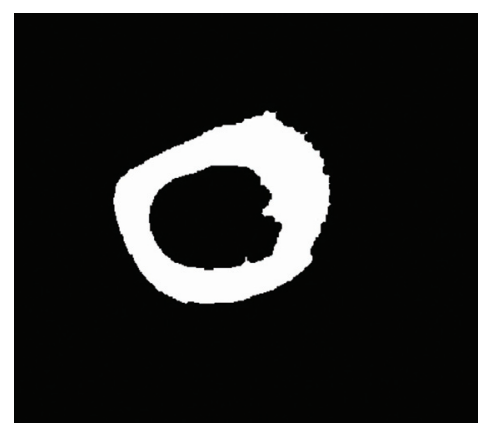

Figura 4. Delimitação da região de interesse contendo o tecido de reparo e o tecido adjacente 

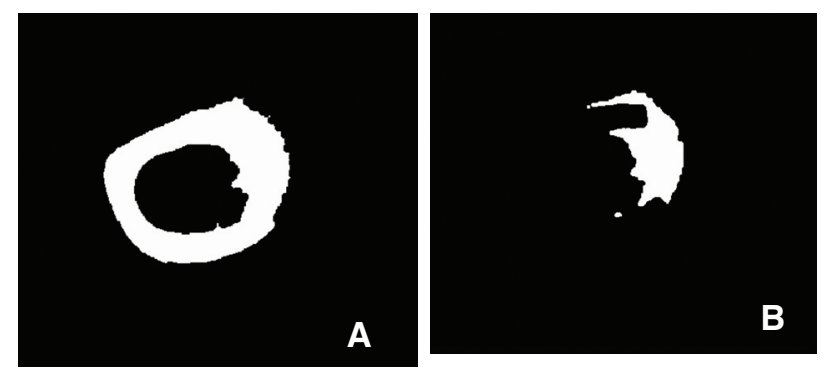

Figura 5. Segmentação do tecido de reparo (B) na região de interesse $(A)$

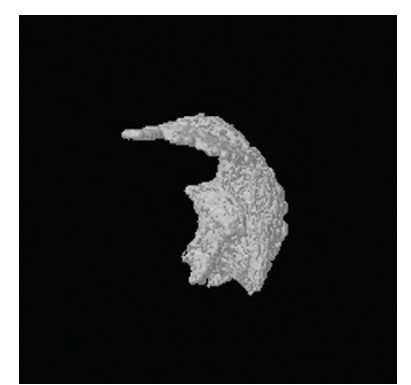

Figura 7. Visualização 3D do tecido de reparo de um fêmur no dia 7

As Figuras 9 e 10 exemplificam a segmentação do tecido de reparo do tecido adjacente em reconstruções microtomográficas de fêmures nos dias 14 e 21 , respectivamente.
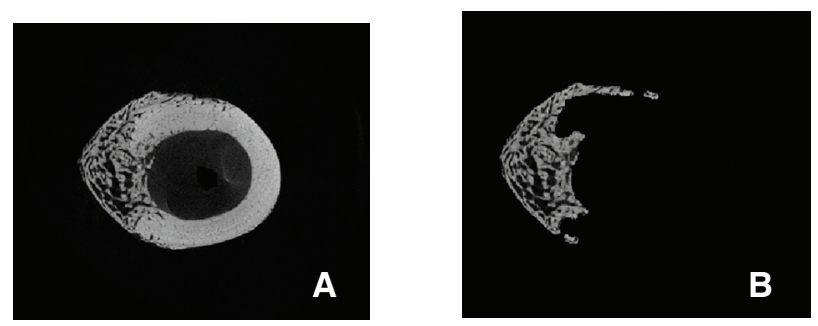

Figura 9. Imagem em tons de cinza da segmentação do tecido de reparo no dia 14 (B) em reconstrução microtomográfica contendo o tecido adjacente $(A)$
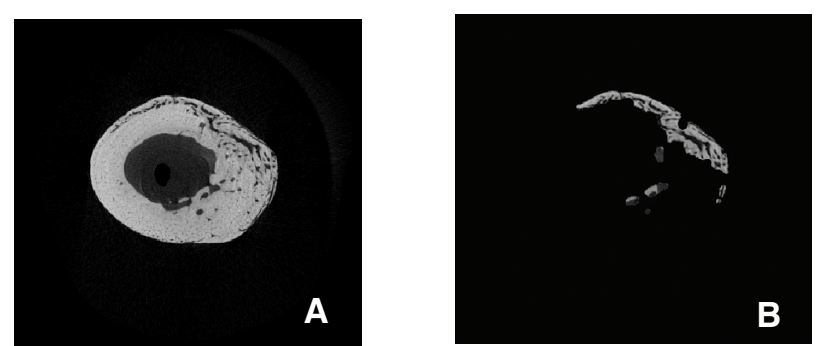

Figura 10. Imagem em tons de cinza da segmentação do tecido de reparo no dia 21 (B) em reconstrução microtomográfica contendo o tecido adjacente $(A)$
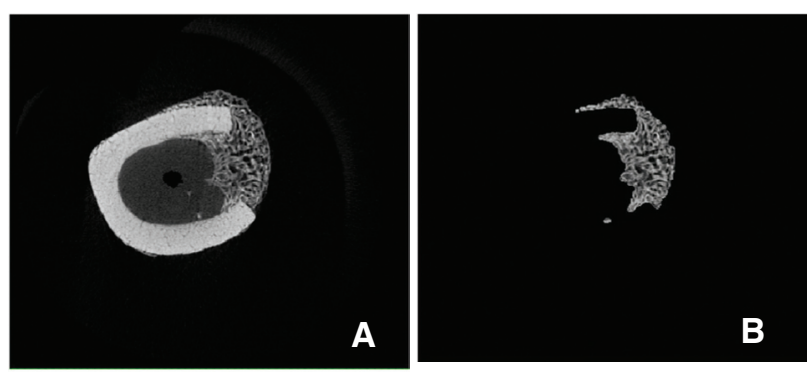

Figura 6. Imagem em tons de cinza da segmentação do tecido de reparo no dia 7 (B) em reconstrução microtomográfica contendo o tecido adjacente $(A)$

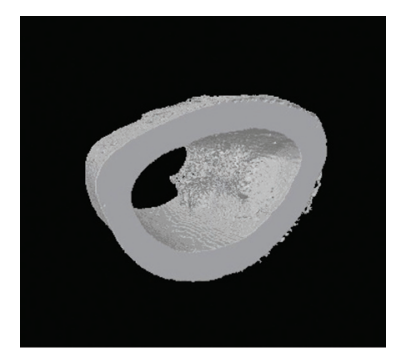

Figura 8. Visualização 3D da região medial do fêmur contendo o tecido de reparo

\section{DISCUSSÃO}

A microtomografia por raio-x possibilita diferentes formas de visualização do reparo ósseo em uma fratura experimental. As visualizações $2 \mathrm{D}$ em diferentes planos e 3D caracterizam uma avaliação qualitativa desse tecido.

$\mathrm{Na}$ segmentação do tecido de reparo ósseo relativa aos dias 7, 14 e 21 (Figuras 6, 9 e 10) observase uma pequena perda de pixels possivelmente causada pelo procedimento de thresholding. Esse procedimento é dependente de uma análise visual pelo usuário do software CT-Analyzer para comparar as reconstruções microtomográficas e o resultado da binarização ao decidir sobre o intervalo de níveis de cinza que serão utilizados no procedimento.

$\mathrm{O}$ algorítimo de processamento de imagem utilizado nessa investigação envolve procedimentos de thresholding, delimitação da região de interesse e segmentação do tecido de reparo do tecido adjacente que antecedem a implementação da quantificação ou análise morfométrica $3 \mathrm{D}$ do tecido de reparo. $A$ análise é realizada pelo mesmo software após a binarização das imagens segmentadas para a quantificação de parâmetros como o volume e a densidade mineral volumétrica óssea. Essa implementação é inerente ao processo em andamento no PPGIB-USP de formação de pesquisadores brasileiros no uso da técnica de microtomografia e de softwares a ela associados para a caracterização do reparo ósseo em fraturas 
Milanetti et al. Avaliação do reparo ósseo por microtomografia por raio-X.

experimentais.

Uma técnica de validação da metodologia de avaliação do reparo ósseo descrita nesta investigação precisa ser desenvolvida. A literatura consultada ${ }^{4-7}$ sobre o uso de uCT para esta avaliação não descreve essa técnica.

A técnica convencional de avaliação do reparo ósseo é a histologia qualitativa que é bidimensional e destrutiva e permite identificar através de corantes específicos a presença de tecidos e células que caracterizam os diversos estágios do reparo. O uso da uCT nessa avaliação possibilita visualizações 2D e $3 D$ da região fraturada e do tecido de reparo, a análise morfométrica 3D de diferentes parâmetros desse tecido e a medida da densidade mineral volumétrica do mesmo. Por ser não destrutiva, após a realização da técnica microtomográfica, o tecido de reparo de ossos fraturados ou osteotomizados podem ser submetidos a outras avaliações, como por exemplo, ensaios para a medida da resistência mecânica. As técnicas histológica e microtomográfica são complementares.

\section{CONCLUSÃO}

A microtomografia por raio-x pode dar importantes contribuições na avaliação do reparo ósseo em fraturas experimentais e têm grande potencial de aplicação em investigações sobre novas tecnologias de tratamento de fraturas.

Agradecimentos: A Coordenação de Aperfeiçoamento de Pessoal de Ensino Superior (CAPES) pelo financiamento de bolsa de mestrado para a realização dessa investigação.

\section{REFERÊNCIAS}

1. Nyman JS, Munhoz S, Jadhav S, Mansour A, Yoshi T, Mundi GR, Gutierrez GE. Quantitative measures of femoral fracture repair in rats derived by micro-computed tomography. J Biomechan. 2009;42(7):891-7.

2. Einhorn TA. The science of fracture healing. J Orthop Trauma. 2005;19(10 Suppl):S4-S6.

3. Augat P, Ryaby JT. Fracture healing and micro architecture. In: Majumdar S, Bay BK, editors. Noninvasive assessment of trabecular bone architecture and the competence of bone. New York: Kluwer/ Plenum Publishers; 2001. p.99-109.

4. Freeman TA, Patel P, Parvizi J, Antoci Jr V, Shapiro IM. Micro-CT analysis with multiple thresholds allows detection of bone formation and resorption during ultrasound-treated fracture healing. J Orthop Res. 2009;27(5):673-9.

5. Morgan EF, Mason ZD, Chien KB, Pfeiffer AJ, Barnes GL, Einhron TA, Gerstenfeld LC. Micro-computed tomography assessment of fracture healing: relationships among callus structure, composition, and mechanical function. Bone. 2009;44(2):335-44.

6. Belleville C, Ceccotti MC, Feyen J. High resolution tomodensitometry of bone fracture healing in mouse. In: Proceedings of Skyscan User Meeting, 15-17 June, Belgium; 2008.

7. Bakker N, Gerrits H, Gossn J. Validation of new potential drugs targets for osteoporosis and fracture repair by in vivo UCT. In: Proceedings of Skyscan User Meeting, 15-17 June, Belgium; 2008.

8. Lirani APR. Estudo comparativo dos efeitos do ultra-som e do laser de baixa intensidade no reparo ósseo de tíbia de ratos [Dissertação]. São Carlos, SP: Programa de Pós-Graduação Interunidades Bioengenharia - USP, Escola de Engenharia de São Carlos, Faculdade de Medicina de Ribeirão Preto, Instituto de Química de São Carlos; 2004.

9. Feldkamp LA, Davis LC, Kress JW. Practical cone-beam algorithm. J Accoust Soc. 1984;1(6):612-9.

Artigo recebido em: 08/02/2011

Artigo aceito em: 15/04/2011 